DOI 10.35381/cm.v6i2.611

\title{
Sistema web adaptativo para mejorar la gestión de ventas en empresas
}

\section{Adaptive web system to improve sales management in companies}

\author{
Luis Albarracín-Zambrano \\ uq.luisalbarracin@uniandes.edu.ec \\ Universidad Regional Autónoma de los Andes, Quevedo \\ Ecuador \\ https://orcid.org/0000-0002-3164-5229 \\ Jonathan Fabián Ponce-Bravo \\ sq.jonathanfpb27@uniandes.edu.ec \\ Universidad Regional Autónoma de los Andes, Quevedo \\ Ecuador \\ https://orcid.org/0000-0002-9855-5455 \\ Mario Aníbal Hidalgo-Fernández \\ sq.marioahf36@uniandes.edu.ec \\ Universidad Regional Autónoma de los Andes, Quevedo \\ Ecuador \\ https://orcid.org/0000-0003-3777-5195 \\ Robert Wladimir Estrada-Apolo \\ sq.robertwea22@uniandes.edu.ec \\ Universidad Regional Autónoma de los Andes, Quevedo \\ Ecuador \\ https://orcid.org/0000-0003-0652-0149
}

Recepción: 15 de septiembre 2021

Aprobación: 15 de noviembre 2021 
Luis Albarracín-Zambrano; Jonathan Fabián Ponce-Bravo; Mario Aníbal Hidalgo-Fernández; Robert Wladimir Estrada-Apolo

\section{Estimado Editor (a):}

Actualmente en Ecuador existen sistemas web especializados en las ventas de materiales eléctricos, como es el caso de INSELEC, el cual se especializa en ofrecer productos y soluciones innovadoras para aplicaciones eléctricas y electrónicas tanto de marcas propias como de terceros, que cumplan los requisitos de diversos sectores y contribuyan a su desarrollo, actúa responsablemente con las personas y el ambiente. En este orden de ideas, Zapata Cortes, Vélez Bedoya y Arango Serna (2020) señalan:

El cumplimiento del nivel de servicio esperado por el cliente, es decir, sus expectativas, son fundamentales para lograr el éxito de las empresas a corto y largo plazo, ya que este cumplimiento permitirá mantener el interés de los clientes por los servicios y bienes de la empresa. (p.5)

Además, la empresa tiene como enfoque fabricar y mantener una constante innovación de los productos marca Beaucoup de acuerdo con los gustos del cliente, bajo normas de calidad nacionales e internacionales. Además, se especializa en comercializar productos electrónicos para usos residenciales, comerciales e industriales.

En este sentido, Santa María Vílchez (2018) manifiesta:

Es importante mencionar que una página Web puede contener elementos que permiten una comunicación activa entre el usuario y la información. Esto permite que el usuario acceda a los datos de modo interactivo, gracias a que la página responderá a cada una de sus acciones. (p.23)

Al respecto, teniendo en consideración la situación que se vive a nivel mundial y nacional por la pandemia del Covid-19. Es de vital importancia que la empresa "DISPROELEC" cuente con un sistema web adaptivo para mejorar sus ventas, facturación y además promocionar sus productos, al respecto Burbano (2018, p. 17) citado por Citado por Paya Villafuerte (2021, p.33) define el sistema:

El diseño adaptativo o responsive es un método que permite modificar la visualización del diseño web para los diferentes dispositivos que existen. "El diseño web responsivo se focaliza para que una página web se pueda ver en cualquier sistema operativo y navegador, para cualquier tamaño de pantalla y 
Luis Albarracín-Zambrano; Jonathan Fabián Ponce-Bravo; Mario Aníbal Hidalgo-Fernández; Robert Wladimir Estrada-Apolo

velocidad de conexión, esos son los tres pilares fundamentales del diseño web responsive.

Así mismo, ya que actualmente el internet es el mejor recurso, representa para el comercio electrónico una herramienta fundamental para el desarrollo de una empresa, dándose a conocer como marca, promociones e eventos en más ciudades del país y porque no fuera del mismo, el sistema web actualmente les permitirá a los empleados con sus respectivas niveles de accesibilidad, llevar registros de clientes, proveedores, stock de productos, gestionar las ventas, facturación, cotizaciones y reportes que se efectúen en la empresa, incrementando así una mejor calidad de servicio hacia el cliente. Internet ofrece importantes oportunidades de negocio para las pequeñas empresas que actúan en el ámbito local o que cuentan con diseños organizativos más simples. (Rodríguez Ardura, Meseguer Artola y Vilaseca Requena,2007, p.98)

Por lo tanto, para Chugnas Morales (2012) indica que:

La gestión de ventas es una relación que se establece entre dos o más personas, en la que el vendedor debe lograr que el cliente se sienta complacido del producto adquirido y del trato recibido. Todo vendedor debe realizar una gestión de venta que engrandezca (p.13)

Es importante manifestar que el sistema Web debe contribuir al proceso constante de mejoramiento, en el cual permitiría la implementación en un futuro del carrito de compras, para brindarle un mejor servicio a los clientes. Además, de funciones en relación a las necesidades que vaya implementando la empresa "DISPROELEC". De allí la importancia de una comunicación constantes con los clientes. En este orden de ideas, Marín Dueñas y Lozano Fernández (2017) manifiestan:

Se puede entender la comunicación comercial empresarial como aquella comunicación que establecen las empresas para relacionarse con los consumidores o personas que influyen en el proceso de compra. Su objetivo básico es lograr el consumo/uso de los productos y servicios que la empresa ofrece al mercado. (p.12) 


\section{FINANCIAMIENTO}

No monetario.

\section{AGRADECIMIENTO}

A la Universidad Regional Autónoma de los Andes, Quevedo, por motivar el desarrollo de la Investigación.

\section{REFERENCIAS CONSULTADAS}

Chugnas Morales, J. (2012). Sistema de gestión de ventas para reducir el tiempo de atención al cliente y aumentar el margen de utilidad en la empresa "CORPORACIÓN DIJOL SRL" en el año 2012. [Sales management system to reduce customer service time and increase profit margin in the company "CORPORACIÓN DIJOL SRL" in 2012]. Tesis de pregrado. Universidad Privada del Norte Laureate International Universities. Recuperado de: https://n9.cl/f7/xo

Marín Dueñas, P., y Lozano Fernández, J. (2017). La comunicación de marketing en la empresa de distribución española Mercadona. [Marketing communication in the Spanish distribution company Mercadona]. RETOS. Revista de Ciencias de la Administración y Economía, 7(13), 9-26. https://doi.org/10.17163/ret.n13.2017.01

Paya Villafuerte Ignacio Melitón (2021) Sistema web para la administración, gestión y control de las ventas e inventarios del taller electromecánico expansión Barros. [Web system for the administration, management and control of sales and inventories of the electromechanical workshop barros expansion].Tesis de pregrado. Universidad Agraria del Ecuador. Milagro. Ecuador. Recuperado de: https://n9.cl/r46rs

Rodríguez Ardura, I., Meseguer Artola, A., y Vilaseca Requena, J. (2007). Sistemas de venta en línea: un análisis de sus factores críticos para el pequeño comerciante. [Online sale systems: an analysis of their critical factors for small business]. Revista de Gestão da Tecnologia e Sistemas de Informação Journal of Information Systems and Technology Management, 4(1),95-108. Recuperado de: https://n9.cl/4ccat 
Santa María Vílchez, C. (2018). Implementación de un sistema web adaptativo para apoyar en la gestión comercial utilizando el método de costo promedio en la empresa Ferrotumi s.a.c. [Implementation of an adaptive web system to support commercial management using the average cost method in the company Ferrotumi s.a.c]. Tesis de Pregrado. Universidad Católica Santo Toribio de Mogrovejo. Chiclayo. Recuperado de: https://n9.cl/8xfmw

Zapata-Cortes, J., Vélez-Bedoya, Á., y Arango-Serna, M. (2020). Mejora del proceso de distribución en una empresa de transporte. [Web system for the administration, management and control of sales and inventories of the electromechanical workshop barros expansion]. Investigación administrativa, 49(126). https://doi.org/10.35426/iav49n126.08

C2021 por los autores. Este artículo es de acceso abierto y distribuido según los términos y condiciones de la licencia Creative Commons Atribución-NoComercial-Compartirlgual 4.0 Internacional (CC BY-NC-SA 4.0) (https://creativecommons.org/licenses/by-nc-sa/4.0/). 